\title{
Forensic species identification of elephant (Elephantidae) and giraffe (Giraffidae) tail hair using light microscopy
}

\author{
Bonnie C. Yates $\cdot$ Edgard O. Espinoza $\cdot$ \\ Barry W. Baker
}

Accepted: 14 May 2010/Published online: 13 June 2010

(C) Springer Science+Business Media, LLC 2010

\begin{abstract}
Here we present methods for distinguishing tail hairs of African elephants (Loxodonta africana), Asian elephants (Elephas maximus), and giraffes (Giraffa camelopardalis) from forensic contexts. Such hairs are commonly used to manufacture jewelry artifacts that are often sold illegally in the international wildlife trade. Tail hairs from these three species are easily confused macroscopically, and morphological methods for distinguishing African and Asian tail hairs have not been published. We used cross section analysis and light microscopy to analyze the tail hair morphology of 18 individual African elephants, 18 Asian elephants, and 40 giraffes. We found that cross-sectional shape, pigment placement, and pigment density are useful morphological features for distinguishing the three species. These observations provide wildlife forensic scientists with an important analytical tool for enforcing legislation and international treaties regulating the trade in elephant parts.
\end{abstract}

Keywords Loxodonta africana - Elephas maximus . Giraffa camelopardalis · Hair identification .

Forensic mammalogy · Wildlife trade $\cdot$ Light microscopy

“Having pull'd out of an Elephants-tayl a black Hair, and cut transversly from it a thin scale, I exposed it to my Microscope, which represented in the thick of that Hair about an hundred little specks somewhat whitish, and in each speck a black point, and in some few

B. C. Yates · E. O. Espinoza - B. W. Baker $(\square)$

US National Fish and Wildlife Forensics Laboratory, United

States Fish \& Wildlife Service, 1490 East Main Street,

Ashland, OR 97520-1310, USA

e-mail: barry_baker@fws.gov of those black points, a little hole; and this hair consisted withal of united Globuls, which yet I thought I should have found bigger in this thick hair of so bulky a Beast, than indeed they were. This Scale I keep still by me because of its curious and elegant appearance, not unlike (excepting the Colours) a Peacocks-tayl."

Anthony van Leeuwenhoek (1674) Philosophical Transactions

"It is an elephant's hair, probably from the tail. But, as you see, it is a compound hair; virtually a group of hairs agglutinated into a single stem."

Dr. Thorndyke (fictional detective) From "The Trail of Behemoth" (1929), by R. Austin Freeman (reprinted, 2004)

\section{Introduction}

Artifacts constructed from elephant and giraffe tail hairs are often encountered in the international wildlife trade (Fig. 1). The most common examples include tail hair bracelets, necklaces, rings and flywhisks used by indigenous peoples and sold to tourists throughout Africa and parts of Southeast Asia [1]. Rigorous methods for identifying elephant and giraffe hairs are of interest to law enforcement officials, who monitor the illegal trade in endangered and threatened species. Tail hairs from all three species are known to be used in artifacts and international trade [1]. Similar artifacts are often manufactured from various plastics and botanical fibers (e.g., elephant grass [Pennisetum purpureum]), thus forensic analysis may be required to determine if such items contain parts of 


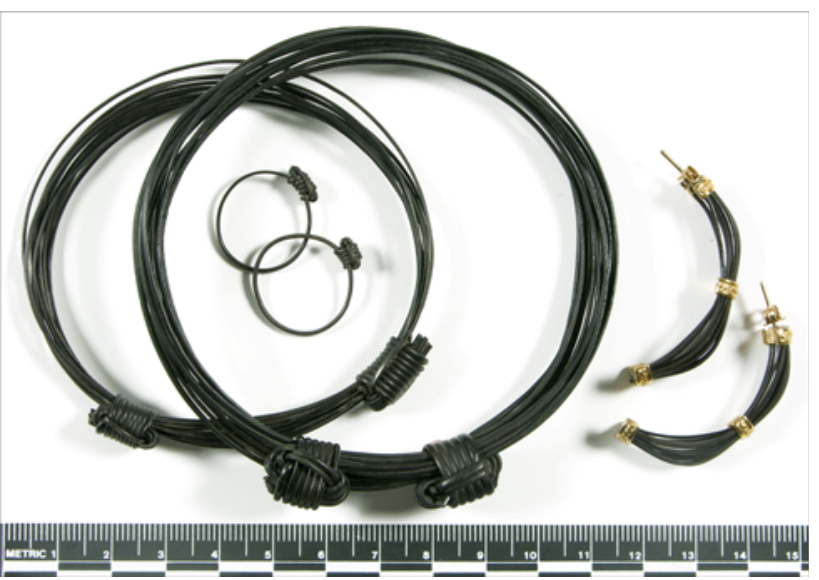

Fig. 1 Examples of elephant hair artifacts, including bracelets, rings and earrings

protected species. While wildlife conservation efforts have focused extensively on the ivory trade, all parts and products manufactured from elephants fall under strict regulatory control and forensic methods for their identification are critical to conservation efforts.

Asian elephants (Elephas maximus) are protected under Appendix I of CITES (Convention on International Trade in Endangered Species of Wild Fauna and Flora), while populations of African elephants (Loxodonta africana) are variously protected under Appendix I and II. The Asian elephant is also listed as endangered on the US Endangered Species Act (ESA), while the African elephant is listed as threatened. In contrast, giraffes (Giraffa camelopardalis) are not protected under CITES or the ESA. Overall, commercial trade in elephant parts is highly regulated, and forensic methods for distinguishing elephant and giraffe tail hairs are critical to wildlife enforcement efforts.

The thick, coarse, black tail hairs of elephants (African and Asian) and giraffes are easily confused macroscopically. While elephant tail hairs are generally thicker than those of giraffe, the size of an individual hair alone cannot be used to distinguish these species. Previous research [1] has reported spectroscopic and chemical differences between the tail hairs of elephants and giraffes, and here we present methods for distinguishing them using cross section morphology and light microscopy. The technique provides a robust means of identifying these fibers with inexpensive, standard laboratory equipment.

\section{Previous microscopic studies}

The earliest microscopic study of elephant tail hair was reported by none other than Anthony van Leeuwenhoek (1674) in a letter to the Royal Society of London [2]. Van Leeuwenhoek's microscopes functioned at up to 266X [3], thus allowing him to describe what he saw in the eloquent language of his time as quoted above. Shoshani [4] described, but did not illustrate, the cross section of an elephant tail hair as "...oval in shape..." and possessing "...a number of small unpigmented areas (white as opposed to the dark color elsewhere in the section)." Microscopic observations on elephant tail hairs have even appeared in a crime novel. Freeman's fictional detective Dr. Thorndyke opined on the identification of a suspected elephant hair recovered from a crime scene, noting "...it is a compound hair; virtually a group of hairs agglutinated into a single stem." [5]. Giraffe tail hairs were described by Lochte [6] as possessing multiple strands of medulla. While several subsequent or earlier studies mention the morphology of elephant and giraffe body hairs, to our knowledge no comparative studies of elephant and giraffe tail hair cross sections have been published in an attempt to distinguish these species.

Among early researchers, Smith [7] illustrated cross sections of skin and body hairs of the elephant, and Neuville [8] noted the variable medullation in elephant hairs, but we are unsure if his study included tail hairs. Hausman [9] included the cuticular structures of elephant, mammoth, and giraffe body hairs. Lochte [10] published what may be the earliest photomicrographs of cross sections and cuticular casts of elephant and giraffe body hairs, which for an Indian (=Asian) elephant are described as fine and thin hair, with no explicit detail. The giraffe hairs were taken from the back near the mane and described as transparent, having no medulla.

Body hairs of these mega mammals and others, such as rhino and hippo, have been described and/or illustrated in several reports [11-16]. Ryder [17] described the follicular mammoth body hairs in detail, but he only illustrated a tracing of a group of hairs from the leg of the Liakhov mammoth. Valente [18] compared mammoth hair from the corpse of a frozen specimen found in 1977 to those of zoo elephants-both Elephas and Loxodonta. He states that the "cortex of Mammuthus hair had tiny dark brown pigment granules and the density of pigmentation decreased from the central axis of the hair to the periphery" [18]. The Elephas hairs were "non-medullated and circular in crosssection... [and] the cortex may or may not contain pigment granules which, if present, are sparse towards the periphery" [18]. By contrast, the body hairs of Loxodonta were "heavily pigmented throughout...except for the region immediately underlying the cuticle" [18].

Rasmussen and Munger [19] show the cross section of a vibrissa in micrographs of skin from the trunk of an Asian elephant, although their paper concerned the sensory innervation of that appendage and not the hairs themselves. A low magnification photomicrograph (50X) of one of these hairs [19] shows an unmedullated, pigmented center 
surrounded by concentric rings of connective tissue that is separated from an outer capsule of connective tissue by an amorphous ring of "vascular sinus spaces." While these previous studies focused on body hair morphology, here we concentrate our analysis on the structural features of elephant and giraffe tail hairs (cross-sectional shape, pigment placement, and density) and the use of these morphological features for identifying the species source of tail hairs in forensic contexts.

\section{Methods}

Vouchered tail hairs of known species origin were examined from the collection of the US National Fish and Wildlife Forensics Laboratory (United States Fish \& Wildlife Service). Samples consisted of 18 individual African elephants (Loxodonta africana), 18 Asian elephants (Elephas maximus), and 40 giraffes (Giraffa camelopardalis) from captive zoo settings. Background data on these vouchered samples were previously published [1]. While the forest elephant is recognized as a full species (Loxodonta cyclotis) by some researchers [20], here we follow CITES taxonomy and other researchers who treat Loxodonta from Africa as a single species (Loxodonta africana) [21].

Hairs were cross-sectioned, examined with light microscopy, and evaluated for species diagnostic characters. Cross section analysis has long been used to evaluate mammal hair morphology and facilitate species identification [22]. Hairs were sonicated in test tubes with tap water for $10 \mathrm{~min}$ to loosen and remove detritus. Water was decanted, and hairs were subsequently sonicated in isopropyl alcohol to remove non-water-soluble particles. Hairs were allowed to air dry. To facilitate cross-sectioning, individual hairs were placed in centrifuge tubes and filled with epoxy to serve as a stabilizing agent (EPO-Kwik resin and hardener by Buehler, Lake Bluff, IL). The filled tubes were allowed to dry/set for $24 \mathrm{~h}$. Once set, centrifuge tubes were placed in a mechanical microtome (Model 860, American Optical Co., Buffalo, NY) and were cross-sectioned ( $\approx 26 \mu$ thick). Samples were mounted onto permanent slides using Shandon E-Z Mount (Thermo Fisher Scientific Inc., Waltham, MA) and were examined and analyzed unstained at 100, 200, and $400 \times$ with a Leica dual-stage transmitted light microscope. Images were produced with LAS software (Leica Microsystems, Inc., Bannockburn, IL).

\section{Results}

Our cross sections reveal that tail hairs of elephants and giraffes differ significantly from body hairs, and suggest that they are more like rhinoceros horn or horse hoof keratins $[23,24]$. To describe them as hairs, one would need to qualify that they appear to have multiple medullae on the interior and generally lack cuticular scales on the exterior. They are more like small, cylindrical segments of thicker keratinous outgrowths of epidermis, comparable to that seen in horn or hoof. As Pollitt described for horses' hooves, "...the keratinocytes of individual hoof wall tubules are arranged around a central hollow medulla in non-pigmented concentric layers" [24]. In a single elephant hair, therefore, these tubules appear to be imbedded in a keratinized cellular matrix [23] confined by the diameter of the tail "hair."

In their expansive SEM study of animal hairs, Chernova and Tselikova [13] published micrographs of cross sections of both mammoth and African elephant hairs. Unlike the fine Asian elephant hair pictured in the early publication by Lochte [10], their electron micrographs of the mammoth and Loxodonta hairs show the same tubule-like features at relatively low magnification that we see in our tail hair specimens.

The dispersion of pigment granules in the matrix is affected by the location of the tubules. The resulting pattern that the granules form defines the characterization that distinguishes each species. Thus, it is both the arrangement of the tubules and the dispersion of the pigment that creates the diagnostic traits identifiable in tail hairs of elephants and giraffe (Figs. 2, 3).

Similar tubules observed in hairs and other keratins have variously been referred to as sinuses [19], vacuoles [25], cortical cells with cavities [26], compressed tubules [27], or tubules [24], which is the preferred term here. Chernova [28] stated there is "impelling need for consistent detailed description of cellular structure" for microscopic features of both human and animal hair. In our study, we found some of these tubules to be empty cavities, and, indeed, in a scanning electron micrograph generated on one of our

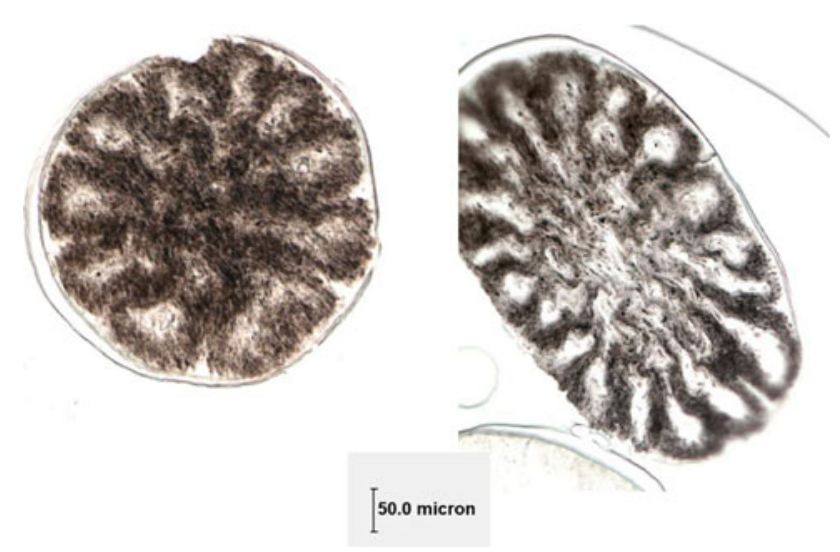

Fig. 2 Cross sections of giraffe tail hairs at $100 \times$ 


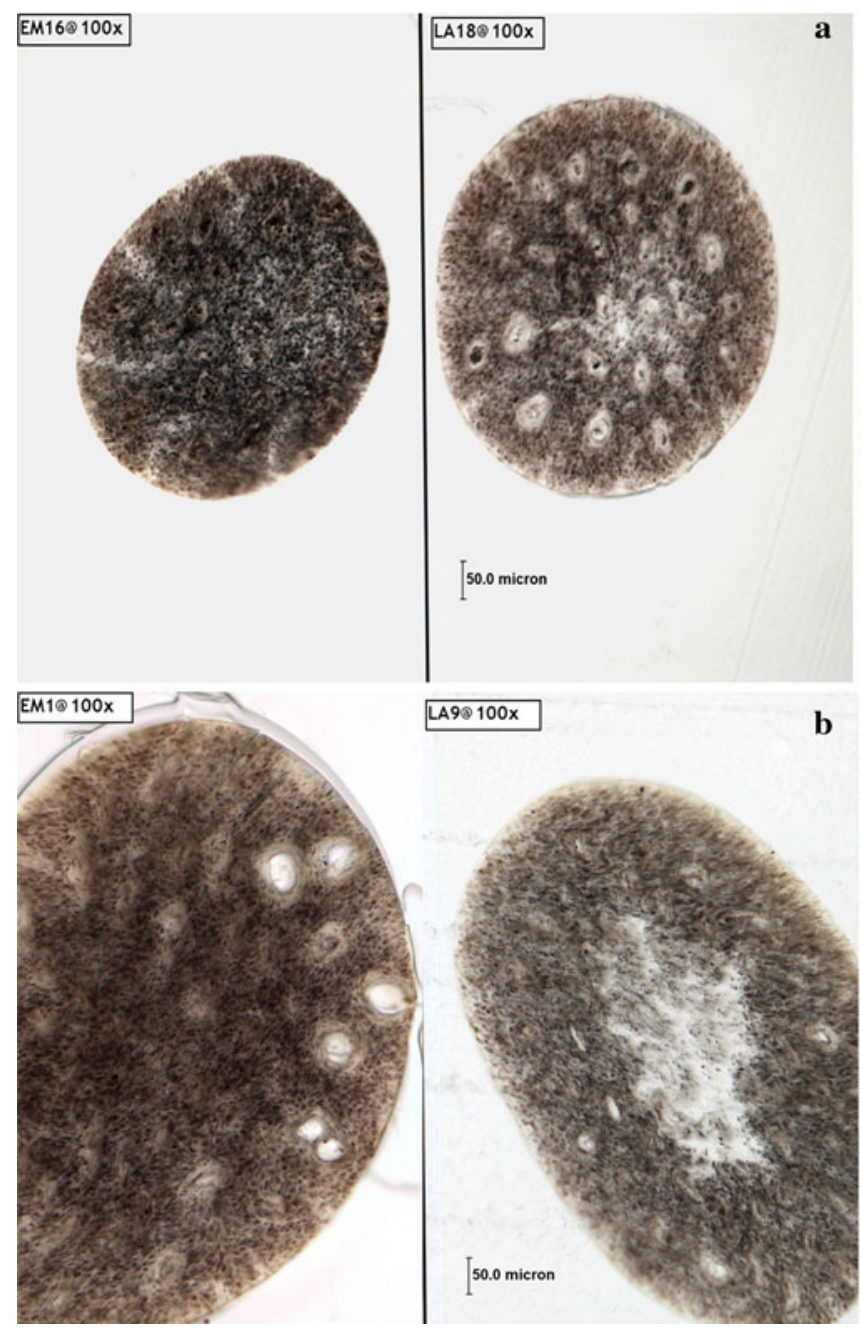

Fig. 3 a Cross section of Asian elephant (left) and African elephant (right) tail hairs at $100 \times$. b Cross section of Asian elephant (left) and African elephant (right) tail hairs at $100 \times$. c Cross section of Asian

specimens (Fig. 4), a remnant of the matrix got caught during sectioning in one of the holes near the periphery.

In other tubules shown in Fig. 4, they are in-filled, either with what appears to be a yellowish matter also visible in the transmitted light micrographs (Fig. 5), or by differentiated keratinous cells retained by the elongating tubule during its growth phase. As these tubules migrate during growth toward the periphery of the hair, the vacuous ones reach the edge of the tail hair and form grooves, which in cross section look like a scallop-shaped edge (Fig. 5). These grooves can be seen in stereomicrographs running the length of the tail hair. We speculate that the different expressions of the tubules (compressed, in-filled, or vacuous,) are related to the morphogenesis and development of keratinocytes that eventually mature and migrate away from the tubule to form the matrix.
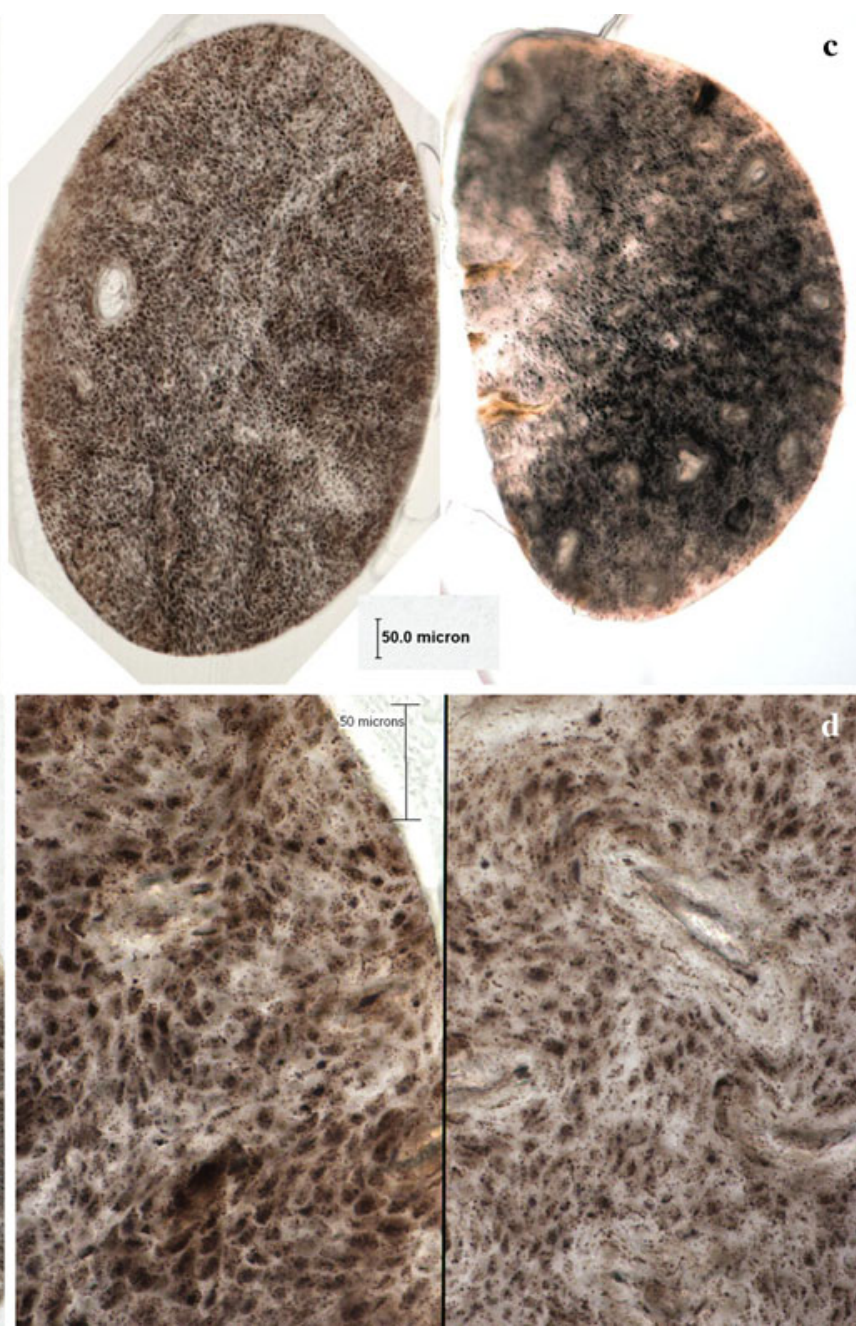

elephant (left) and African elephant (right) tail hairs at $100 \times$. d Cross section of Asian elephant (left) and African elephant (right) tail hairs at $400 \times$

\section{Distinguishing traits}

Giraffe vs. elephant

The character trait that distinguishes giraffe from elephant (both African and Asian) is the distinct radial arrangement (in giraffe) of the tubules toward the periphery of the hair shaft (Fig. 2; Table 1). They appear as oval or flattened tubules arranged in a ring positioned more than two-thirds the radius of the tail hair's diameter from its center. The pigment is usually densest in-between the tubules and appears to flow away from the un-pigmented keratin cells that surround the formed or forming tubules. The effect is a pattern of undulating un-pigmented rays on a dark mottled ground, emanating from the center of the cross section and forming regularly spaced, dark-centered tubules surrounded 


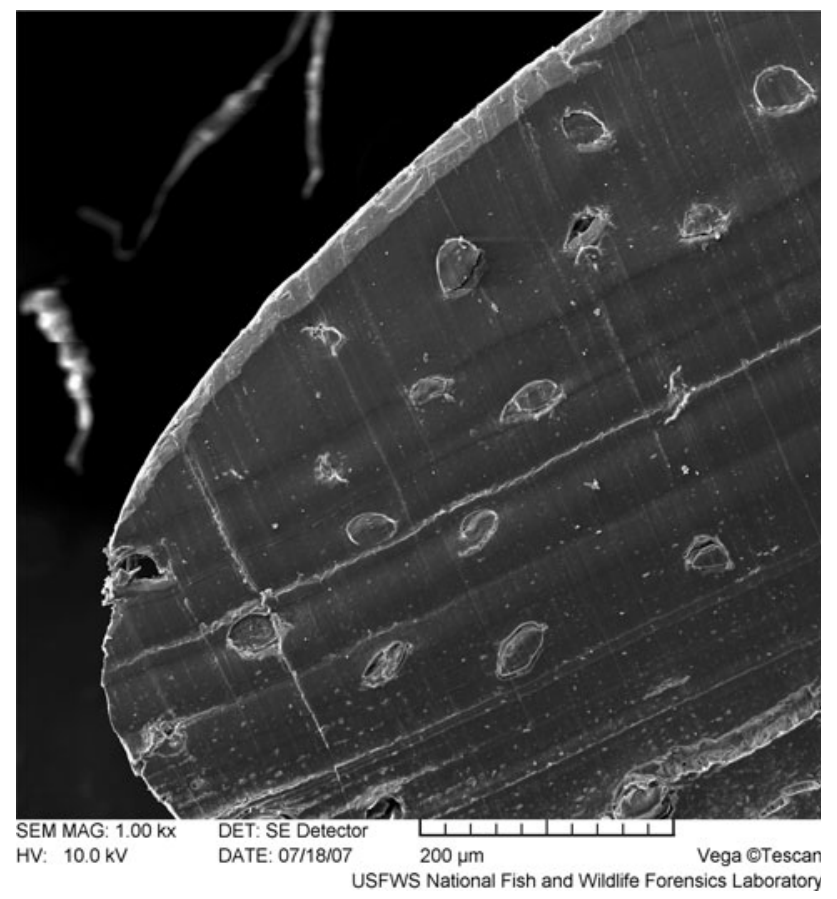

Fig. 4 SEM of Asian elephant tail hair at 1,000×

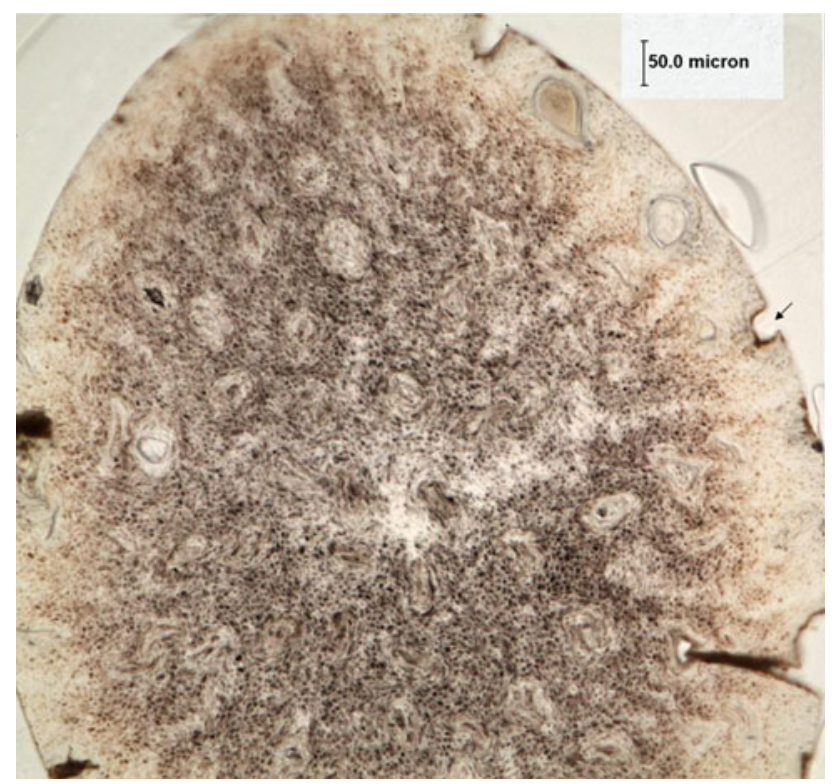

Fig. 5 Cross section of African elephant tail hair at $100 \times$

by un-pigmented concentric wavy rings, which are the maturing keratinocytes of the tubules' walls.

As noted previously, the structure of these hairs is reminiscent of horse hoof keratin [24], but on a much smaller scale. Unlike horse's hoof, the tubules in tail hair are weaker and, therefore, appear less organized and more variable in shape, and because they comprise hair, the generation of the tubules is centered in the cylinder and grows outward from there. In proximal sections taken from giraffe tail hairs, the tubules have not begun to migrate toward the periphery, but still appear to be organized in the less pigmented center, trending toward the outside wall where the pigment granules are darkest, but where fingers of un-pigmented space have intruded and are beginning to follow the progression of the tubule and its attendant un-pigmented cells toward the periphery (Fig. 2).

\section{African vs. Asian elephant}

The character traits that distinguish African elephant (Loxodonta) from Asian elephant (Elephas) are the density and appearance of the pigment assemblages, and the arrangement of the tubules (Table 1). The two elephant species can be distinguished by the larger, darker pigment granules and denser assemblages in the keratin matrix of Elephas, and by the more diffuse, blurry-appearing assemblages in Loxodonta (Fig. 3d). While the large granules are about the same size in both elephants, there appears to be fewer large granules in Loxodonta. In both elephants' tail hairs, rings of undifferentiated keratinocytes form around the tubules, which may be vacuous or infilled or compressed, but in Loxodonta, the rings conform to tubules that are more polygonal in shape, numbering up to 50 in some cross sections. By contrast, the rings around the tubules in Elephas are more rounded in shape and do not influence the arrangement of nearby pigment granules as seen in Loxodonta. In Elephas, there are fewer tubules, and the rings are less distinct and fainter in appearance than those of Loxodonta.

Finally, there is a noticeable absence of pigmentation that is expressed differently in each species. For example, cross sections of Loxodonta tail hairs frequently are almost void of pigmentation in the center, while Elephas is usually heavily pigmented throughout. In addition, Elephas exhibits the ray-like features more or less absent of pigment granules that are common to Giraffa, though fewer in number, but like Giraffa tend to radiate from the center.

\section{Conclusions}

Our research into thickened tail hairs of elephants and giraffes was driven by the occurrence of these animal parts in the international wildlife trade. To date, morphological cross-section comparison of the tail hairs of these species was lacking. Our light microscopy analysis of tail hairs shows that cross-sectional shape, pigment placement, and pigment density are useful morphological features for distinguishing these three species. While we do not yet know of a functional explanation for these differences, we have demonstrated that these species can be distinguished using inexpensive and standard laboratory equipment. 
Table 1 Summary of microscopic traits that distinguish cross sections of Elephas, Loxodonta, and Giraffa tail hairs

\begin{tabular}{llll}
\hline & Giraffe & Asian elephant & African elephant \\
\hline Pigment & Dark \& dense & Dark and dense & Few dark aggregates \\
& & & Blurry fine aggregates \\
Tubules* & Usually infilled & May be infilled or empty & May be infilled or empty \\
& Equidistant when near periphery & Scattered unevenly & Rarely found in center, which also lacks pigment aggr. \\
"Rays"** & Trail from tubules back to center & Few in number, but present & Rare \\
\hline
\end{tabular}

* Vacuities in the field of keratin that are ovoid or compressed

** Undulating streaks devoid of pigment aggregates

These results compliment previous research demonstrating chemical differences between elephant and giraffe tail hairs [1]. These methods should prove useful for enforcing legislation and international treaties regulating the trade in elephant parts.

\section{Key points}

1. Light microscopy is a useful tool for identifying the species origin of elephant and giraffe tail hairs in forensic contexts.

2. This study describes the first reported morphological differences between the tail hairs of African elephants and Asian elephants.

3. Tail hairs from the three species under investigation can be identified based on differences in cross-sectional shape, pigment placement, and pigment density.

4. This study compliments previous research documenting chemical differences between elephant and giraffe tail hairs.

Acknowledgments Doina Voin provided German and Russian translations of relevant literature and helped acquire the tail hairs used in this study. Laura Blount prepared the hair cross sections and mounted the hairs used in this study. Darby Morrell produced Fig. 1 and Michael Scanlan produced Fig. 4. The Sterling Evans Library at Texas A\&M University was instrumental in obtaining literature. We thank Linzi Wilson-Wilde for inviting us to submit this manuscript. The findings and conclusions in this article are those of the authors and do not necessarily represent the views of the U.S. Fish and Wildlife Service.

\section{References}

1. Espinoza EO, Baker BW, Moores TD, Voin D. Forensic identification of elephant and giraffe hair artifacts using HATR FTIR spectroscopy and discriminant analysis. Endangered Species Res. 2008;9:239-46.

2. Leeuwenhoek Mr. Microscopical observations from Mr. Leeuwenhoeck, about blood, milk, bones, the brain, spittle, cuticula, sweat, fatt, teares; communicated in two letters to the publisher. Phil Trans 1674;(1665-1678)9:121-31.
3. Van Zuylen J. The microscopes of Antoni van Leeuwenhoek. J Microsc. 1981;121:309-28.

4. Shoshani J, et al. On the dissection of a female Asian elephant (Elephas maximus maximus Linnaeus, 1758) and data from other elephants. Elephant. 1982;2:3-93.

5. Freeman RA. The famous cases of Dr. Thorndyke: thirty-seven of his criminal investigations, Part 2. Kessinger Publishing; 2004.

6. Lochte T. Das mikroskopische bild des giraffen hares. Zool Garten. 1952;9:204-6.

7. Smith F. The histology of the skin of the elephant. J Anat Physiol. 1890;24:493-503.

8. Neuville MH. Du tégument des proboscidiens. Bull Mus Hist Nat Paris. 1917;23:374-87.

9. Hausman LA. Structural characteristics of the hair of mammals. Am Nat. 1920;54:496-523.

10. Lochte T. Atlas der Menschlichen und Tierischen Haare. Germany: Leipzig; 1938.

11. Buys D, Hillary JK. Notes on the microstructure of hair of the Orycteropodidae, Elephantidae, Equidae, Suidae and Giraffidae. S Afr J Wildl Res. 1984;14:111-9.

12. Cave AJE. Hairs and vibrissae in the Rhinocerotidae. J Zool. 1968;157:247-57.

13. Chernova OF, Tselikova TN. An atlas of mammalian hair: fine structure of overhair and hair using scanning electron microscopy. Moscow: KMK Sci Press; 2004. In Russian.

14. Dimond RL, Montagna W. The skin of the giraffe. Anat Rec. 1976;185:63-75.

15. Hammond EC, Jones C. An examination of a strand of 30,000 and 70,000 year old mammoth hair. In: Bailey GW, Bentley J, Small JA, editors. Proceedings of the 50th Annual Meeting of the Electron Microscopy Society of America, San Francisco, CA: Electron Microscopy Soc; 1992, vol 50, no 2, pp. 1100-1101.

16. Kranz KR. A note on the structure of tail hairs from a pygmy hippopotamus (Choeropsis liberiensis). Zoo Biol. 1982;1: 237-41.

17. Ryder ML. Hair of the mammoth. Nature. 1974;249:190-2.

18. Valente A. Hair structure of the woolly mammoth, Mammuthus primigenius and the modern elephants, Elephas maximus and Loxodonta africana. J Zool. 1983;199:271-4.

19. Rasmussen LEL, Munger BL. The sensorineural specializations of the trunk tip (finger) of the Asian elephant, Elephas maximus. Anat Rec. 1996;246:127-34.

20. Roca AL, Georgiadis N, O'Brien SJ. Cyto-nuclear genomic dissociation and the African elephant species question. Q Int. 2007;169-70:4-16.

21. Johnson MB, Clifford SL, Goossens B, Nyakaana S, Curran B, White LJT, Wickings EJ, Bruford MW. Complex phylogeographic history of central African forest elephants and its implications for taxonomy. BMC Evol Biol. 2007;7:244.

22. Williams CS. A simple method for sectioning mammalian hairs for identification purposes. J Mammal. 1934;15:251-2. 
23. Hieronymus TL, Witmer LM, Ridgely RC. Structure of white rhinoceros (Ceratotherium simum) horn investigated by $\mathrm{x}$-ray computed tomography and histology with implications for growth and external form. J Morphol. 2006;267:1172-6.

24. Pollitt C. Anatomy and physiology of the inner hoof wall. Clin Tech Equine Prac. 2004;3:3-21.

25. Clement JL, Hagege R, Le Pareaux A, Carteaud JP. Ultrastructural study of the medulla of mammalian hairs. Scan Electron Microsc. 1981;3:377-82.
26. Sato H, Miyasaka S, Yoshino M, Seta S. Morphological comparison of the cross section of the human and animal hair shafts by scanning electron microscopy. Scan Electron Microsc. 1982;1:115-25.

27. Blakey PR, Lockwood P. The environment of calcified components in keratins. Calcif Tissue Res. 1968;2:361-9.

28. Chernova OF. Architectonics of the medulla of guard hair and its importance for identification of taxa. Doklady Biol Sci. 2001;376:81-5. 